\title{
On the Geometry of Curves in Minkowski 3-Space and Its Foldings
}

\author{
A. E. El-Ahmady ${ }^{1,2}$, E. Al-Hesiny ${ }^{1}$ \\ ${ }^{1}$ Mathematics Department, Faculty of Science, Taibah University, Madinah, KSA \\ ${ }^{2}$ Mathematics Department, Faculty of Science, Tanta University, Tanta, Egypt \\ Email: a_elahmady@hotmail.com, e-1.e-1@hotmail.com
}

Received November 13, 2012; revised April 4, 2013; accepted April 12, 2013

Copyright (C) 2013 A. E. El-Ahmady, E. Al-Hesiny. This is an open access article distributed under the Creative Commons Attribution License, which permits unrestricted use, distribution, and reproduction in any medium, provided the original work is properly cited.

\begin{abstract}
We will introduce a new connection between some transformations and some aspects of differential geometry of some curves in Minkowski space. The concept of folding, retractions and contraction on some curves in Minkowski space will be characterized by using some aspects of differential geometry. Types of the deformation retracts of some curves in Minkowski 3-space are obtained. The relations between the foldings and the deformation retracts of some curves are deduced. The connections between some transformations and time like, space like, light like of some curves in Minkowski 3-space are also presented.
\end{abstract}

Keywords: Retractions; Deformation Retracts; Folding; Contraction; Minkowski 3-Space

\section{Introduction and Definitions}

As is well known, the theory of deformation retract is always one of the interesting topics in Euclidian and Non-Euclidian space and it has been investigated from the various viewpoints by many branches of topology and differential geometry El-Ahmady [1-3].

Minkowski space is originally from the relativity in physics. In fact, a time like curve corresponds to the path of an observer moving at less than the speed of light, a light like curve corresponds to moving at the speed of light and a space like curve moving faster than light El-Ahmady [4,5].

The Minkowski 3-space $E_{1}^{3}$ is the Euclidean 3-space $E_{1}^{3}$ provided with the standard flat metric given by $g=-d x_{1}^{2}+d x_{2}^{2}+d x_{3}^{2}$, where $\left(x_{1}, x_{2}, x_{3}\right)$ is a rectangular coordinate system of $E_{1}^{3}$. Since $g$ is an indefinite metric, recall that a vector $v \in E_{1}^{3}$ can have one of three Lorentzian causal characters, it can be space like if $g(v, v)>$ 0 or $v=0$, time like if $g(v, v)<0$ and light like if $g(v, v)=0$ and $v \neq 0$. Similarly, an arbitrary curve $\alpha=\alpha(s)$ in $E_{1}^{3}$ can locally be space like, time like or light like, if all of its velocity vectors $\alpha^{\prime}(s)$ are respectively, space like, time like or light like respectively. A curve in Lorentzian space $L^{n}$ is a smooth map $\alpha: I \rightarrow L^{n}$, where $I$ is the open interval in the real line $\mathbb{R}$. The interval has a coordinate system consisting of the identity map $u$ of $I$. The velocity of $\alpha$ at $t \in I$ is $\alpha^{\prime}=\left.\frac{\mathrm{d} \alpha(u)}{\mathrm{d} u}\right|_{t}$.

A curve $\alpha$ is said to be regular if $\alpha^{\prime}(s)$ does not vanish for all $t \in I . \alpha \in L^{n}$ is space like if its velocity vectors $\alpha^{\prime}(t)$ are space like for all $t \in I$, similarly for timelike and null. If $\alpha$ is a null curve, we can reparametrize it such that $\left\langle\alpha^{\prime}(t), \alpha^{\prime}(t)\right\rangle=0$ and $\alpha^{\prime}(t) \neq 0$ El-Ahmady [5].

Most folding problems are attractive from a pure mathematical standpoint, for the beauty of the problems themselves. The folding problems have close connections to important industrial applications. Linkage folding has applications in robotics and hydraulic tube bending. Paper folding has application in sheet-metal bending, packaging, and air-bag folding El-Ahmady [6]. Following the great Soviet geometer El-Ahmady [5], also, used folding to solve difficult problems related to shell structures in civil engineering and aero space design, namely buckling instability El-Ahmady [7]. Isometric folding between two Riemannian manifold may be characterized as maps that send piecewise geodesic segments to a piecewise geodesic segments of the same length $[8,9]$. For a topological folding the maps do not preserves lengths El-Ahmady [8-10] i.e. A map $\mathfrak{I}: M \rightarrow N$, where $M$ and $N$ are $C^{\infty}$-Riemannian manifolds of dimension $m, n$ respectively is said to be an isometric folding of $M$ into $N$, iff 
for any piecewise geodesic path $\gamma: J \rightarrow M$, the induced path $\mathfrak{J} \circ \gamma: J \rightarrow N$ is a piecewise geodesic and of the same length as $\gamma$. If $\mathfrak{I}$ does not preserve length, then $\mathfrak{I}$ is a topological folding El-Ahmady [10-12].

A subset $A$ of a topological space $X$ is called a retract of $X$ if there exists a continuous map $r: X \rightarrow A$ such that $r(a)=a, \forall a \in A$, where $A$ is closed and $X$ is open. El-Ahmady [7-12] and Gregory [13]. This can be restated as follows. If $i: A \rightarrow X$ is the inclusion map, then $r: X \rightarrow A$ is a map such that $r i=i d_{A}$ Miles [14] and Martin [15]. If, in addition $r i \simeq i d_{x}$, we call $r$ a deformation retract and $A$ a deformation retract of $X$ Jeffrey [16] and John [17].

\section{Main Result}

Let $\alpha(s)$ be a curve in the space-time $E_{1}^{3}$, parameterized by arc length function $s$ Lopez [18] and Formiga [19]. Then for the unit speed curve $\alpha(s)$ with non-null frame vectors the following Frenet equations are given in

$$
\left[\begin{array}{l}
T^{\prime} \\
N^{\prime} \\
B^{\prime}
\end{array}\right]=\left[\begin{array}{ccc}
0 & k & 0 \\
\varepsilon_{1} k & 0 & \varepsilon_{2} \tau \\
0 & \varepsilon_{3} \tau & 0
\end{array}\right]\left[\begin{array}{l}
T \\
N \\
B
\end{array}\right]
$$

We write following subcases.

1) If $\alpha(s)$ is space-like curve in $E_{1}^{3}$, then $T$ is a space-like vector. Thus, we distinguish according to $N$.

Case 1: If $N$ is space-like vector, then $B$ is time-like vector, then $\varepsilon_{i}(1 \leq i \leq 3)$ read $\varepsilon_{1}=-1, \varepsilon_{2}=\varepsilon_{3}=1$. And $T, N$ and $B$ are mutually orthogonal vectors satisfying equations, $g(T, T)=g(N, N)=1, g(B, B)=-1$.

Case 2: $N$ is time-like vector, then $\varepsilon_{i}(1 \leq i \leq 3)$ read $\varepsilon_{1}=\varepsilon_{2}=\varepsilon_{3}=1$.

And $T, N$ and $B$ are mutually orthogonal vectors satisfying equations

$$
g(T, T)=g(B, B)=1, g(N, N)=-1 .
$$

2) If $\alpha(s)$ is time-like curve in $E_{1}^{3}$, then $T$ is timelike vector. Then $\varepsilon_{i}(1 \leq i \leq 3)$ read $\varepsilon_{1}=\varepsilon_{2}=1, \varepsilon_{3}=-1$. And $T, N$ and $B$ are mutually orthogonal vectors satisfying equations,

$$
g(N, N)=g(B, B)=1, g(T, T)=-1 .
$$

3) If $\alpha(s)$ is light-like curve in $E_{1}^{3}$, then the following Frenet equations are given in

$$
\left[\begin{array}{c}
T^{\prime} \\
N^{\prime} \\
B^{\prime}
\end{array}\right]=\left[\begin{array}{ccc}
0 & 1 & 0 \\
\tau & 0 & -1 \\
0 & -\tau & 0
\end{array}\right]\left[\begin{array}{l}
T \\
N \\
B
\end{array}\right]
$$

Also, if $\alpha(s)$ be a curve in the space-time $E_{1}^{4}$, parameterized by arc length function $s$. Then for the unit speed curve $\alpha(s)$ with non-null frame vectors the fol- lowing Frenet equations are given in

$$
\left[\begin{array}{c}
T^{\prime} \\
N^{\prime} \\
B_{1}^{\prime} \\
B_{2}^{\prime}
\end{array}\right]=\left[\begin{array}{cccc}
0 & k & 0 & 0 \\
\mu_{1} k & 0 & \mu_{2} \tau & 0 \\
0 & \mu_{3} \tau & 0 & \mu_{4} \sigma \\
0 & 0 & \mu_{5} \sigma & 0
\end{array}\right]\left[\begin{array}{c}
T \\
N \\
B_{1} \\
B_{2}
\end{array}\right]
$$

Due to character of $\alpha(s)$, we write following subcases.

1) If $\alpha(s)$ is space-like curve in $E_{1}^{4}$, then $T$ is a space-like vector. Thus, we distinguish according to $N$.

Case 1: If $N$ is space-like vector, then $B_{1}$ can have two causal characters.

Case 1.1: $B_{1}$ is space-like vector, then $\mu_{i}(1 \leq i \leq 5)$ read, $\mu_{1}=\mu_{3}=-1, \mu_{2}=\mu_{4}=\mu_{5}=1$. And $T, N, B_{1}$ and $B_{2}$ are mutually orthogonal vectors satisfying equations

$$
g(T, T)=g(N, N)=g\left(B_{1}, B_{1}\right)=1, g\left(B_{2}, B_{2}\right)=-1
$$

Case 1.2: $B_{1}$ is time-like vector, then $\mu_{i}(1 \leq i \leq 5)$ read $\mu_{1}=-1, \mu_{2}=\mu_{3}=\mu_{4}=\mu_{5}=1$. And $T, N, B_{1}$ and $B_{2}$ are mutually orthogonal vectors satisfying equations

$$
g(T, T)=g(N, N)=g\left(B_{2}, B_{2}\right)=1, g\left(B_{1}, B_{1}\right)=-1
$$

Case 2: $N$ is time-like vector, then $\mu_{i}(1 \leq i \leq 5)$ read $\mu_{1}=\mu_{2}=\mu_{3}=\mu_{4}=1, \mu_{5}=-1$. And $T, N, B_{1}$ and $B_{2}$ are mutually orthogonal vectors satisfying equations

$$
g(T, T)=g\left(B_{1}, B_{1}\right)=g\left(B_{2}, B_{2}\right)=1, g(N, N)=-1 .
$$

2) If $\alpha(s)$ is time-like curve in $E_{1}^{4}$, then $T$ is timelike vector. Then $\mu_{i}(1 \leq i \leq 5)$ read $\mu_{1}=\mu_{2}=\mu_{4}=1$, $\mu_{3}=\mu_{5}=-1$. And $T, N, B_{1}$ and $B_{2}$ are mutually orthogonal vectors satisfying equations

$$
g(N, N)=g\left(B_{1}, B_{1}\right)=g\left(B_{2}, B_{2}\right)=1, g(T, T)=-1
$$

Hence, we can formulate the following theorems.

Theorem 1. Under the retraction, a spacelike curve and a timelike curve $r(\alpha(s))$ in the space-time $E_{1}^{3}$ parameterized by arc length function $s$, where $\operatorname{dim} r(\alpha(s))>0$ and with non-vanishing curvature, lies in ahyperplane if and only if the torsion vanishes identically.

Proof. Suppose the curve $r(\alpha(s))$ lies in a hyperplane. Let us assume that we can bring $r(\alpha(s))$ to lie in the $\left(x^{0}, x^{1}, 0\right)$-hyperplane. Then the parametric equations of $r(\alpha(s))$ are of the form $x^{a}(s)=\left(x^{0}(s), x^{1}(s), 0\right)$. Let $\left(e_{0}, e_{1}, e_{2}\right)$ denote the vectors of the canonical coordinate basis. Thus, in these coordinates,

$$
T=\frac{\mathrm{d} x^{\alpha}(s)}{\mathrm{d} s}=\frac{\mathrm{d} x^{0}}{\mathrm{~d} s} e_{0}+\frac{\mathrm{d} x^{1}}{\mathrm{~d} s} e_{1}
$$

and

$$
\frac{\mathrm{d} T}{\mathrm{~d} s}=\frac{\mathrm{d}^{2} x^{\alpha}(s)}{\mathrm{d} s^{2}}=\frac{\mathrm{d}^{2} x^{0}}{\mathrm{~d} s^{2}} e_{0}+\frac{\mathrm{d}^{2} x^{1}}{\mathrm{~d} s^{2}} e_{1} .
$$


From (1) we have $\frac{\mathrm{d} T}{\mathrm{~d} s}=K N$. Since $k \neq 0$ then $N$ has no components in the $x^{2}$-direction, i.e.

$$
N=\left(y^{0}(s), y^{1}(s), 0\right) \text {. }
$$

Thus, $\frac{\mathrm{d} N}{\mathrm{~d} s}=\frac{\mathrm{d} y^{0}}{\mathrm{~d} s} e_{0}+\frac{\mathrm{d} y^{1}}{\mathrm{~d} s} e_{1}$ hence from the equation $\frac{\mathrm{d} N}{\mathrm{~d} s}=\varepsilon_{1} k T+\varepsilon_{2} \tau B$ we conclude that $\tau \mathrm{z}^{2}(s)=0$, where $B=\left(z^{0}(s), z^{1}(s), z^{2}(s)\right)$. However, $z^{2}(s)$ cannot be zero. Otherwise the set of vectors $\{T, N, B\}$ would not be linearly independent. Then $\tau$ must vanish.

Suppose that $\tau=0$. Since $\frac{\mathrm{d} B}{\mathrm{~d} s}=\varepsilon_{2} \tau N$, then $B$ is a constant vector. Let us conveniently choose our coordinate system in such a way that $e_{2}=B$. Now, since $T$ is orthogonal to $B$ we must have $B \cdot T=0$, which means that $r(\alpha(s))$ lies in the hyperplane $x^{2}(s)=$ const .

Corollary 1. Under the folding, $f: \alpha(s) \rightarrow \alpha(s)$, a spacelike curve and a timelike curve $f(\alpha(s))$ in the space-time $E_{1}^{3}$ parametrized by arc length function $s$, with non-vanishing curvature, lies in a hyperplane if and only if the torsion vanishes identically.

Theorem 2. Under the retraction, if the curve is a lightlike curve $r(\alpha(s))$ in the space-time $E_{1}^{3}$ parameterized by arc length function $s$, where

$\operatorname{dim} r(\alpha(s))>0$ then the curve is not lies in a hyperplane.

Proof. Suppose the curve $r(\alpha(s))$ lies in a hyperplane. Let us assume that we can bring $r(\alpha(s))$ to lie in the $\left(x^{0}, x^{1}, 0\right)$-hyperplane. Then the parametric equations of $r(\alpha(s))$ are of the form $x^{\alpha}(s)=\left(x^{0}(s), x^{1}(s), 0\right)$. Let $\left(e_{0}, e_{1}, e_{2}\right)$ denote the vectors of the canonical coordinate basis. Thus, in these coordinates,

and

$$
T=\frac{\mathrm{d} x^{\alpha}(s)}{\mathrm{d} s}=\frac{\mathrm{d} x^{0}}{\mathrm{~d} s} e_{0}+\frac{\mathrm{d} x^{1}}{\mathrm{~d} s} e_{1}
$$

$$
\frac{\mathrm{d} T}{\mathrm{~d} s}=\frac{d^{2} x^{\alpha}(s)}{\mathrm{d} s^{2}}=\frac{\mathrm{d}^{2} x^{0}}{\mathrm{~d} s^{2}} e_{0}+\frac{\mathrm{d}^{2} x^{1}}{\mathrm{ds} s^{2}} e_{1} .
$$

From (2) we have $\frac{\mathrm{d} T}{\mathrm{~d} s}=N$. Then $N$ has no components in the $x^{2}$-direction, i.e. $N=\left(y^{0}(s), y^{1}(s), 0\right)$. Thus, $\frac{\mathrm{d} N}{\mathrm{~d} s}=\frac{\mathrm{d} y^{0}}{\mathrm{~d} s} e_{0}+\frac{\mathrm{d} y^{1}}{\mathrm{~d} s} e_{1}$, hence from the equation $\frac{\mathrm{d} N}{\mathrm{~d} s}=\tau T-B$, we conclude $z^{2}(s)=0$, where

$B=\left(z^{0}(s), z^{1}(s), z^{2}(s)\right)$. But $z^{2}(s)$ cannot be zero because the set of vectors $\{T, N, B\}$ would not be linearly independent, then the curve is not lies in a hyper- plane.

Corollary 2. Under the folding, $f: \alpha(s) \rightarrow \alpha(s)$ if the curve is a lightlike curve $f(\alpha(s))$ in the spacetime $E_{1}^{3}$ parameterized by arc length function $s$, then the curve is not lies in a hyperplane.

Theorem 3. Under the retraction, a spacelike curve and a timelike curve $r(\alpha(s))$ in the space-time $E_{1}^{4}$ parameterized by arc length function $s$, where $\operatorname{dim} r(\alpha(s))>0$ and with non-vanishing curvature, lies in ahyperplane if and only if the second torsion vanishes identically.

Proof. Let us start with the necessary condition. Suppose the curve $r(\alpha(s))$ lies in a hyperplane. Let us assume that we can bring $r(\alpha(s))$ to lie in the $\left(x^{0}, x^{1}, x^{2}\right)$-hyperplane. Then the parametric equations of $r(\alpha(s))$ are of the form

$x^{\alpha}(s)=\left(x^{0}(s), x^{1}(s), x^{2}(s), 0\right)$. Let $\left(e_{0}, e_{1}, e_{2}, e_{3}\right)$ denote the vectors of the canonical coordinate basis. Thus, in these coordinates,

and

$$
T=\frac{\mathrm{d} x^{\alpha}(s)}{\mathrm{d} s}=\frac{\mathrm{d} x^{0}}{\mathrm{~d} s} e_{0}+\frac{\mathrm{d} x^{1}}{\mathrm{~d} s} e_{1}+\frac{\mathrm{d} x^{2}}{\mathrm{~d} s} e_{2}
$$

$$
\frac{\mathrm{d} T}{\mathrm{~d} s}=\frac{\mathrm{d}^{2} x^{\alpha}(s)}{\mathrm{d} s^{2}}=\frac{\mathrm{d}^{2} x^{0}}{\mathrm{~d} s^{2}} e_{0}+\frac{\mathrm{d}^{2} x^{1}}{\mathrm{~d} s^{2}} e_{1}+\frac{\mathrm{d}^{2} x^{2}}{\mathrm{~d} s^{2}} e_{2} .
$$

From (3) we have $\frac{\mathrm{d} T}{\mathrm{~d} s}=N$. Since $k \neq 0$ then $N$ has no components in the $x^{3}$-direction, i.e.

$$
N=\left(y^{0}(s), y^{1}(s), y^{2}(s), 0\right) .
$$

Thus, $\frac{\mathrm{d} N}{\mathrm{~d} s}=\frac{\mathrm{d} y^{0}}{\mathrm{~d} s} e_{0}+\frac{\mathrm{d} y^{1}}{\mathrm{~d} s} e_{1}+\frac{\mathrm{d} y^{2}}{\mathrm{~d} s} e_{2}$, hence from the equation $\frac{\mathrm{d} N}{\mathrm{~d} s}=\mu_{1} k T+\mu_{2} \tau B_{1}$ and we conclude that $\tau z^{3}(s)=0$, where $B_{1}=\left(z^{0}(s), z^{1}(s), z^{2}(s), z^{3}(s)\right)$. If $\tau=0$, then $\sigma$ also must vanish, for in this case $B_{1}$ is chosen to be constant. If $\tau \neq 0$, then $z^{3}(s)=0$, hence $B_{1}=\left(z^{0}(s), z^{1}(s), z^{2}(s), 0\right)$. Also,

$\frac{\mathrm{d} B_{1}}{\mathrm{~d} s}=\frac{\mathrm{d} z^{0}}{\mathrm{~d} s} e_{0}+\frac{\mathrm{d} z^{1}}{\mathrm{~d} s} e_{1}+\frac{\mathrm{d} z^{2}}{\mathrm{~d} s} e_{2}$ and the third Serret-Frenet equation $\frac{\mathrm{d} B_{1}}{\mathrm{~d} s}=\mu_{3} \tau N-\mu_{4} \sigma B_{2}$ we are led to conclude that $\sigma \varpi^{3}(s)=0$, where

$$
B_{2}=\left(\varpi^{0}(s), \varpi^{1}(s), \varpi^{2}(s), \varpi^{3}(s)\right) .
$$

However, $\varpi^{3}(s)$ cannot be zero. Otherwise the set of vectors $\left\{T, N, B_{1}, B_{2}\right\}$ would not be linearly independent. Then $\sigma$ must vanish.

Suppose that $\sigma=0$. Since $\frac{\mathrm{d} B_{2}}{\mathrm{~d} s}=\mu_{5} \sigma B_{1}$, then $B_{2}$ is a constant vector. Let us conveniently choose our coordinate system in such a way that $e_{3}=B_{2}$. Now, since 
$T$ is orthogonal to $B_{2}$ we must have $B_{2} \cdot T=0$, which means that $r(\alpha(s))$ lies in the hyperplane $x^{3}(s)=$ const .

Corollary 3. Under the contraction, a spacelike curve and a timelike curve $C(\alpha(s))$ in the space-time $E_{1}^{4}$ parameterized by arc length function $s$, where

$\operatorname{dim} C(\alpha(s))>0$ and with non-vanishing curvature, is lies in a hyperplane if the first and second torsions vanishes identically.

Corollary 4. Under the folding, a spacelike curve and a timelike curve $f(\alpha(s))$ in the space-time $E_{1}^{4}$ parameterized by arc length function $s$, with non-vanishing curvature, lies in a hyperplane if and only if the second torsion vanishes identically.

Corollary 5. Under the folding, $f: \alpha(s) \rightarrow \alpha(s)$, a spacelike curve and a timelike curve $f(\alpha(s))$ in the space-time $E_{1}^{4}$ parameterized by arclength function $s$, with non-vanishing curvature, is lies in a hyperplane if the first and second torsions vanishes identically.
Theorem 4. Given differentiable functions $K(s)>0$ and $\tau(s), s \in I$ such that $s$ is the arc length, there exists a regular parameterized spacelike curve under the folding with the spacelike vector $N, f(\alpha(s))$, in the space-time $E_{1}^{3}$. Also, $K(s)$ is the curvature and $\tau(s)$ is the torsion of $f(\alpha(s))$. Moreover, any other spacelike curve $\bar{\alpha}(s)$ with the spacelike vector $\bar{N}$ satisfying the same conditions and $f\left(\alpha\left(s_{0}\right)\right)=\bar{\alpha}\left(s_{0}\right)$ at $s_{0} \in I$ then $f(\alpha(s))=\bar{\alpha}(s)$ and the Frenet trihedrons of $f(\alpha(s))$ and $\bar{\alpha}(s)$ is identically.

Proof. Now, assume that two curves $f(\alpha)=f(\alpha(s))$ and $\bar{\alpha}=\bar{\alpha}(s)$ satisfy the conditions $k(s)=\bar{k}(s)$ and $\tau(s)=\bar{\tau}(s), s \in I$. Let $T_{0}, N_{0}, B_{0}$ and $\bar{T}_{0}, \bar{N}_{0}, \bar{B}_{0}$ be the Frenet trihedrons of $f(\alpha(s))$ and $\bar{\alpha}(s)$ at $s_{0} \in I$, respectively. Since $f\left(\alpha\left(s_{0}\right)\right)=\bar{\alpha}\left(s_{0}\right)$ then $T_{0}=\bar{T}$, $N_{0}=\bar{N}$ and $B_{0}=\bar{B}$, where $T(s), N(s), B(s)$ and $\bar{T}(s), \bar{N}(s), \bar{B}(s)$ are the Frenet trihedrons of $f(\alpha(s))$ and $\bar{\alpha}(s) \forall s \in I$, respectively. We now observe, by using the Frenet equations at (1), that

$$
\begin{aligned}
& \frac{\mathrm{d}}{\mathrm{d} s}\left\{|T(s)-\bar{T}(s)|^{2}+|N(s)-\bar{N}(s)|^{2}+|B(s)-\bar{B}(s)|^{2}\right\} \\
= & \frac{\mathrm{d}}{\mathrm{d} s}\{\langle T(s)-\bar{T}(s), T(s)-\bar{T}(s)\rangle+\langle N(s)-\bar{N}(s), N(s)-\bar{N}(s)\rangle+\langle B(s)-\bar{B}(s), B(s)-\bar{B}(s)\rangle\} \\
= & 2\left\langle T(s)-\bar{T}(s), T^{\prime}(s)-\bar{T}^{\prime}(s)\right\rangle+2\left\langle N(s)-\bar{N}(s), N^{\prime}(s)-\bar{N}^{\prime}(s)\right\rangle+2\left\langle B(s)-\bar{B}(s), B^{\prime}(s)-\bar{B}^{\prime}(s)\right\rangle \\
= & 2\langle T(s)-\bar{T}(s), k N(s)-k \bar{N}(s)\rangle+2\left\langle N(s)-\bar{N}(s), \varepsilon_{1} K T(s)-\varepsilon_{2} \tau B(s)-\varepsilon_{1} K \bar{T}(s)+\varepsilon_{2} \tau \bar{B}(s)\right\rangle \\
& +2\left\langle B(s)-\bar{B}(s), \varepsilon_{3} \tau N(s)-\varepsilon_{2} \tau \bar{N}(s)\right\rangle \\
= & 2\langle T(s)-\bar{T}(s), k N(s)-k \bar{N}(s)\rangle+2\left\langle N(s)-\bar{N}(s), \varepsilon_{1} k T(s) \varepsilon_{1} k \bar{T}(s)\right\rangle \\
& -2\left\langle N(s)-\bar{N}(s), \varepsilon_{1} \tau B(s)-\varepsilon_{2} \tau \bar{B}(s)\right\rangle+2\left\langle B(s)-\bar{B}(s), \varepsilon_{3} \tau N(s)-\varepsilon_{3} \tau \bar{N}(s)\right\rangle=0
\end{aligned}
$$

for all $s \in I$. Thus, the above expression is constant, and, since it is zero for $s=s_{0}$, it is identically zero. It follows that $T(s)=\bar{T}(s), N(s)=\bar{N}(s), B(s)=\bar{B}(s)$ for all $s \in I$.

$$
\text { Since } \frac{\mathrm{d} \alpha}{\mathrm{d} s}=T(s)=\bar{T}(s)=\frac{\mathrm{d} \bar{\alpha}}{\mathrm{d} s} \text {, we obtain } \frac{\mathrm{d}}{\mathrm{d} s}(\alpha-\bar{\alpha})=0 \text {. }
$$

Thus $\alpha(s)=\bar{\alpha}(s)+a$, where $a$ is a constant vector. Since $\alpha\left(s_{0}\right)=\bar{\alpha}\left(s_{0}\right)$, we have $a=0$; hence, $\alpha(s)=$ $\bar{\alpha}(s)$ for all $s \in I$.

Corollary 6. Given differentiable functions $k(s)>0$ and $\tau(s), s \in I$ such that $s$ is the arc length, there exists a regular parameterized spacelike curve under the contraction with the spacelike vector $N, C(\alpha(s))$, in the space-time $E_{1}^{3}$. Also, $k(s)$ is the curvature and $\tau(s)$ is the torsion of $C(\alpha(s))$. Moreover, any other spacelike curve $\bar{\alpha}(s)$ with the spacelike vector $\bar{N}$ satisfying the same conditions and $C\left(\alpha\left(s_{0}\right)\right)=\bar{\alpha}\left(s_{0}\right)$ at $s_{0} \in I$ then $C(\alpha(s))=\bar{\alpha}(s)$ and the Frenet trihedrons of $C(\alpha(s))$ and $\bar{\alpha}(s)$ is identically.

Theorem 5. Given differentiable functions $k(s)>0$, $\tau(s)$ and $\sigma(s), s \in I$ such that $s$ is the arc length, there exists a regular parameterized spacelike curve under the folding with the spacelike vectors $N$ and $B_{1}$, $f(\alpha(s))$ in the space-time $E_{1}^{4}$. Also, $k(s)$ is the curvature, $\tau(s)$ is the first torsion, and $\sigma(s)$ is the second torsion of $f(\alpha(s))$. Moreover, any other spacelike curve $\bar{\alpha}(s)$ with the spacelike vectors $\bar{N}$ and $\bar{B}_{1}$ satisfying the same conditions and $f\left(\alpha\left(s_{0}\right)\right)=\bar{\alpha}\left(s_{0}\right)$, where $s_{0} \in I$ then $f(\alpha(s))=\bar{\alpha}(s)$ and the FrenetSerret formulas of $f(\alpha(s))$ and $\bar{\alpha}(s)$ is identically.

Proof. Now assume that two curves $f(\alpha)=f(\alpha(s))$ and $\bar{\alpha}=\bar{\alpha}(s)$ satisfy the conditions $K(s)=\bar{K}(s)$, $\tau(s)=\bar{\tau}(s)$ and $\sigma(s)=\bar{\sigma}(s), s \in I$. Let $T_{0}, N_{0}$, $B_{1_{0}}, B_{2_{0}}$ and $\bar{T}_{0}, \bar{N}_{0}, \bar{B}_{1_{0}}, \bar{B}_{2_{0}}$ be the Frenet-Serret formulas of $f(\alpha(s))$ and $\bar{\alpha}(s)$ at $s_{0} \in I$, respectively. Since $f\left(\alpha\left(s_{0}\right)\right)=\bar{\alpha}\left(s_{0}\right)$ then $T_{0}=\bar{T}_{0}, N_{0}=\bar{N}_{0}$, $B_{1_{0}}=\bar{B}_{1_{0}}$, and $B_{2_{0}}=\bar{B}_{2_{0}}$. And $T(s), N(s), B_{1}(s)$, $B_{2}(s)$ and $\bar{T}(s), \bar{N}(s), \bar{B}_{1}(s), \bar{B}_{2}(s)$ are the Frenet-Serret formulas of $f(\alpha(s))$ and $\bar{\alpha}(s) \forall s \in I$, respectively. We now observe, by using the Frenet equations at (3), that 


$$
\begin{aligned}
\frac{\mathrm{d}}{\mathrm{d} s}\left\{|T(s)-\bar{T}(s)|^{2}+|N(s)-\bar{N}(s)|^{2}+\left|B_{1}(s)-\bar{B}_{1}(s)\right|^{2}+\left|B_{2}(s)-\bar{B}_{2}(s)\right|^{2}\right\} \\
=\frac{\mathrm{d}}{\mathrm{d} s}\{\langle T(s)-\bar{T}(s), T(s)-\bar{T}(s)\rangle+\langle N(s)-\bar{N}(s), N(s)-\bar{N}(s)\rangle \\
\left.\quad+\left\langle B_{1}(s)-\bar{B}_{1}(s), B_{1}(s)-\bar{B}_{1}(s)\right\rangle+\left\langle B_{2}(s)-\bar{B}_{2}(s), B_{2}(s)-\bar{B}_{2}(s)\right\rangle\right\} \\
=2\left\langle T(s)-\bar{T}(s), T^{\prime}(s)-\bar{T}^{\prime}(s)\right\rangle+2\left\langle N(s)-\bar{N}(s), N^{\prime}(s)-\bar{N}^{\prime}(s)\right\rangle \\
\quad+2\left\langle B_{1}(s)-\bar{B}_{1}(s), B_{1}^{\prime}(s)-\bar{B}_{1}^{\prime}(s)\right\rangle+2\left\langle B_{2}(s)-\bar{B}_{2}(s), B_{2}^{\prime}(s)-\bar{B}_{2}^{\prime}(s)\right\rangle \\
=2\langle T(s)-\bar{T}(s), K N(s)-K \bar{N}(s)\rangle+2\left\langle N(s)-\bar{N}(s),-K T(s)+\tau B_{1}(s)+K \bar{T}(s)-\tau \bar{B}_{1}(s)\right\rangle \\
\quad+2\left\langle B_{1}(s)-\bar{B}_{1}(s),-\tau N(s)-\sigma B_{2}(s)+\tau \bar{N}(s)+\sigma \bar{B}_{2}(s)\right\rangle+2\left\langle B_{2}(s)-\bar{B}_{2}(s), \sigma B_{1}(s)-\sigma B_{1}(s)\right\rangle \\
=2 k\langle T(s)-\bar{T}(s), N(s)-\bar{N}(s)\rangle-2 k\langle N(s)-\bar{N}(s), T(s)-\bar{T}(s)\rangle+2 \tau\left\langle N(s)-\bar{N}(s), B_{1}(s)-\bar{B}_{1}(s)\right\rangle \\
\quad-2 \tau\left\langle B_{1}(s)-\bar{B}_{1}(s), N(s)-\bar{N}(s)\right\rangle-2 \sigma\left\langle B_{1}(s)-\bar{B}_{1}(s), B_{2}(s)-\bar{B}_{2}(s)\right\rangle \\
\quad+2 \sigma\left\langle B_{2}(s)-\bar{B}_{2}(s), B_{1}(s)-\bar{B}_{1}(s)\right\rangle=0
\end{aligned}
$$

for all $s \in I$. Thus, the above expression is constant and, since it is zero for $s=s_{0}$, it is identically zero. It follows that

$$
\begin{aligned}
& T(s)=\bar{T}(s), N(s)=\bar{N}(s), \\
& B_{1}(s)=\bar{B}_{1}(s), B_{2}(s)=\bar{B}_{2}(s)
\end{aligned}
$$

for all $s \in I$. Since $\frac{\mathrm{d} \alpha}{\mathrm{d} s}=T(s)=\bar{T}(s)=\frac{\mathrm{d} \bar{\alpha}}{\mathrm{d} s}$. We obtain $\frac{\mathrm{d}}{\mathrm{d} s}(\alpha(s)-\bar{\alpha}(s))=0$. Thus $\alpha(s)=\bar{\alpha}(s)+a$, where $a$ is a constant vector. Since $\alpha\left(s_{0}\right)=\bar{\alpha}\left(s_{0}\right)$, we have $a=0$; hence, $\alpha(s)=\bar{\alpha}(s)$ for all $s \in I$.

Theorem 6. Given differentiable functions $k(s)>0$, $\tau(s)$ and $\sigma(s), s \in I$ such that $s$ is the arc length, there exists a regular parameterized spacelike curve under the deformation retract with the spacelike vectors $N$ and $B_{1}, D(\alpha(s))$, in the space-time $E_{1}^{4}$. Also, $k(s)$ is the curvature, $\tau(s)$ is the first torsion, and $\sigma(s)$ is the second torsion of $D(\alpha(s))$. Moreover, any other spacelike curve $\bar{\alpha}(s)$ with the spacelike vectors $\bar{N}$ and $\bar{B}_{1}$ satisfying the same conditions and $D\left(\alpha\left(s_{0}\right)\right)=\bar{\alpha}\left(s_{0}\right)$ where $s_{0} \in I$ then $D(\alpha(s))=\bar{\alpha}(s)$ and the Frenet-Serret formulas of $D(\alpha(s))$ and $\bar{\alpha}(s)$ is identically.

Theorem 7. Let $f(\alpha(s))$ be a simple closed hyperplane curve under the folding in $E_{1}^{3}$ with length $l$, and let $A$ be the area of the region bounded by $f(\alpha(s))$. Then

$$
l^{2}-4 \pi A \geq 0
$$

and equality holds if and only if $f(\alpha(s))$ is a circle.

Proof. Let $E$ and $E^{\prime}$ be two parallel lines which do not meet the closed curve $f(\alpha(s))$ and moves them together until they first meet $f(\alpha(s))$. We thus obtain two parallel tangent lines to $f(\alpha(s)), L$ and $L^{\prime}$, so that the curve is entirely contained in the strip bounded by $L$ and $L^{\prime}$. Consider a circle $S^{1}$ which is tangent to both $L$ and $L^{\prime}$ and does not meet $f(\alpha(s))$. Let $O$ be the center of $S^{1}$ and take a coordinate system with origin at $O$ and the $x$ axis perpendicular to $L$ and $L^{\prime}$. Parameterize $f(\alpha(s))$ by arc length, since $f(\alpha(s))$ simple closed a hyperplane curve, then $f(\alpha(s))=(x(s), y(s), 0)$, so that it is positively oriented and the tangency points of $L$ and $L^{\prime}$ are $s=0$ and $s=s_{1}$, respectively. We can assume that the equation of $S^{1}$ is $\bar{\alpha}(s)=(\bar{x}(s), \bar{y}(s), 0)=(x(s), \bar{y}(s), 0), s \in[0,1]$, where $2 r$ is the distance between $L$ and $L^{\prime}$. Denoting by $\bar{A}$ the area bounded by $S^{1}$, we have

$$
A=\int_{0}^{l} x \dot{y}^{\prime} \mathrm{d} s, \bar{A}=\pi r^{2}=-\int_{0}^{l} \bar{y} \dot{x}^{\prime} \mathrm{d} s
$$

thus

$$
\begin{aligned}
A+\pi r^{2} & =\int_{0}^{l}\left(x \dot{y}^{\prime}-\bar{y} x^{\prime}\right) \mathrm{d} s \leq \int_{0}^{l} \sqrt{\left(x \dot{y}^{\prime}-\bar{y} x^{\prime}\right)^{2}} \mathrm{~d} s \\
& \leq \int_{0}^{l} \sqrt{\left(x^{2}+\bar{y}^{2}\right)\left(\left(x^{\prime}\right)^{2}+\left(\dot{y}^{\prime}\right)^{2}\right)} \mathrm{d} s \\
& =\int_{0}^{l} \sqrt{\left(x^{2}+\bar{y}^{2}\right)} \mathrm{d} s=l r
\end{aligned}
$$

We now notice the fact that the geometric mean of two positive numbers is smaller than or equal to their arithmetic mean, and equality holds if and only if they are equal. It follows that

$$
\sqrt{A} \sqrt{\pi r^{2}} \leq \frac{1}{2}\left(A+\pi r^{2}\right) \leq \frac{1}{2} l r
$$

Therefore, $4 A \pi r^{2} \leq l^{2} r^{2}$ then $l^{2}-4 \pi A \geq 0$.

Now, assume that equality holds in Equation (4). Then equality must hold everywhere in Equations (5) and (6). From the equality in Equation (6) it follows that $A=\pi r^{2}$. Thus, $l=2 \pi r$ and $r$ does not depend on the choice of 
the direction of $L$. Furthermore, equality in Equation (5) implies that $\left(x \dot{y}^{\prime}-\bar{y} x^{\prime}\right)^{2}=\left(x^{2}-\bar{y}^{2}\right)\left(\left(x^{\prime}\right)^{2}+\left(\dot{y}^{\prime}\right)^{2}\right)$, or $\left(x x^{\prime}+\bar{y} y^{\prime}\right)^{2}=0$; that is,

$$
\begin{gathered}
\frac{x}{y^{\prime}}=-\frac{\bar{y}}{x^{\prime}} \\
\frac{\sqrt{x^{2}+\bar{y}^{2}}}{\sqrt{\left(x^{\prime}\right)^{2}+\left(\dot{y}^{\prime}\right)^{2}}}= \pm r \text {. thus, } x= \pm r y^{\prime}
\end{gathered}
$$

Since $r$ does not depend on the choice of the direction of $L$, we can interchange $x$ and $y$ in the last relation and obtain $y= \pm r x^{\prime}$. Thus,

$x^{2}+y^{2}=r^{2}\left(\left(x^{\prime}\right)^{2}+\left(\dot{y}^{\prime}\right)^{2}\right)=r^{2}$ and $f(\alpha(s))$ is a circle.

Theorem 8. Let $D(\alpha(s))$ be a simple closed a hyperplane curve under the deformation retract in $E_{1}^{3}$ with length $l$, and let $A$ be the area of the region bounded by $D(\alpha(s))$. Then $l^{2}-4 \pi A \geq 0$, and equality holds if and only if $r(\alpha(s))$ is a circle.

Corollary 7. Let $C(\alpha(s))$ be a simple closed a hyperplane curve under the contraction in $E_{1}^{3}$ with length $l$, and let $A$ be the area of the region bounded by $C(\alpha(s))$. Then $l^{2}-4 \pi A \geq 0$, and equality holds if and only if $C(\alpha(s))$ is a circle.

Any $n$ vectors forming a basis for $E_{1}^{n}$ will be written $e_{1}, e_{2}, \cdots, e_{n}$, i.e. the basis will be written $\left(e_{i}\right)$. Relative to a basis $\left(e_{i}\right)$, any vector $\lambda$ in $E_{1}^{n}$ is uniquely expressible in the form

$$
\begin{gathered}
\lambda=\lambda^{1} e_{1}+\lambda^{2} e_{2}+\cdots+\lambda^{n} e_{n} \\
\lambda=\sum_{i=1}^{n} \lambda^{i} e_{i}
\end{gathered}
$$

The numbers $\lambda^{i}$, where $i=1,2, \cdots, n$, are called the components of $\lambda$ relative to the basis $\left(e_{i}\right)$. If $\mu^{i}$ are the components of another vector $\mu$ relative to the same basis $\mu=\sum_{i=1}^{n} \mu^{i} e_{i}$. Let the vectors $e_{1^{\prime}}, e_{2^{\prime}}, \cdots, e_{n^{\prime}}$ form another basis of $E_{1}^{n}$. Since each vector $e_{i^{\prime}}$ is uniquely expressible as a linear combination of the vectors $\left(e_{i}\right)$, we have

$$
e_{i^{\prime}}=\sum_{i=1}^{n} \boldsymbol{p}_{i^{\prime}}^{i} e_{i}
$$

where $\left(\boldsymbol{p}_{i^{\prime}}^{i}\right)$ is an $n \times n$ matrix, non-singular because the vectors $e_{i}$, are linearly independent. Similarly, the vector $e_{i^{\prime}}$ is uniquely expressible in the form

$$
e_{i}=\sum_{i^{\prime}=1}^{n} \boldsymbol{p}_{i}^{i^{\prime}} e_{i^{\prime}}
$$

where $\left(\boldsymbol{p}_{i}^{i^{\prime}}\right)$ is a non-singular $n \times n$ matrix. Then

$$
e_{i}=\sum_{i^{\prime}=1}^{n} \boldsymbol{p}_{i}^{i^{\prime}} e_{i^{\prime}}=\sum_{i^{\prime}, j=1}^{n} \boldsymbol{p}_{i}^{i^{\prime}} \boldsymbol{p}_{i^{\prime}}^{j} e_{j}
$$

The linear independence of the basis vectors implies that $\boldsymbol{p}_{i}^{i^{\prime}} \boldsymbol{p}_{i^{\prime}}^{j}=\delta_{i}^{j}$, where $\delta_{i}^{j}$ called the kroneckel delta, takes the value 1 if $i=j$ and is otherwise zero.

Theorem 9. The components of a vector $\lambda$ in $E_{1}^{n}$ where defined relative to the basis $\left(e_{i}\right)$, and a change of basis will induce a change of components.

Proof. The law of transformation for the components of the vector $\lambda$ will now be found when the basis is change from $\left(e_{i}\right)$ to $\left(e_{i^{\prime}}\right)$ according the Equation (7). If the vector $\lambda$ has components $\lambda^{i}$ relative to the basis $\left(e_{i}\right)$, it is convenient to write $\lambda^{i^{\prime}}$ for it's components relative to the new basis $\left(e_{i^{\prime}}\right)$, related to the former by (7). Then

$$
\lambda=\sum_{i=1}^{n} \lambda^{i} e_{i}=\sum_{I^{\prime}=1}^{n} \lambda^{i^{\prime}} e_{i^{\prime}}
$$

Equations (7), (9) give

$$
\sum_{i=1}^{n} \lambda^{i} e_{i}=\sum_{i, i^{\prime}=1}^{n} \lambda^{i^{\prime}} \boldsymbol{p}_{i^{i}}^{i} e_{i}
$$

From which, since the basis vectors $\left(e_{i}\right)$ are linearly independent, it follows that

$$
\lambda^{i}=\boldsymbol{p}_{i^{i}}^{i} \lambda^{i^{\prime}}
$$

Similarly, substitute in (9) for $e_{i}$ from (8) to get

$$
\sum_{i, i^{\prime}=1}^{n} \lambda^{i} \boldsymbol{p}_{i}^{i^{\prime}} e_{i^{\prime}}=\sum_{i^{\prime}=1}^{n} \lambda^{i^{\prime}} e_{i^{\prime}}
$$

From which, since the basis vectors $\left(e_{i^{\prime}}\right)$ are linearly independent

$$
\lambda^{i^{\prime}}=\boldsymbol{p}_{i}^{i^{\prime}} \lambda^{i}
$$

The Equation (11) expresses the new components in terms of the old component, while Equation (10) expresses the old components in terms of the new component.

Theorem 10. Under the retraction, given differentiable functions $k(s)>0, \tau(s)$ and $\sigma(s)$, there exists a retraction of regular parameterized timelike curve $\alpha(s)$, $r(\alpha(s))$, such that $k(s)$ is the curvature, $\tau(s)$ and $\sigma(s)$ are, respectively, the first and second torsion of $r(\alpha(s))$. Any other curve $\bar{\alpha}(s)$ satisfying the same conditions, different from $r(\alpha(s))$. By a Poincaré transformation.

Proof. Let us assume that two timelike curves $r(\alpha(s))$ and $\bar{\alpha}(s)$ satisfy the conditions $k(s)=\bar{k}(s)$, $\tau(s)=\bar{\tau}(s)$ and $\sigma(s)=\bar{\sigma}(s)$, with $s \in I$, where $I$ is an open interval of $\mathbb{R}$, and $\bar{k}(s), \bar{\tau}(s)$ and $\bar{\sigma}(s)$ are, respectively, the curvature, first and second torsion of $\bar{\alpha}(s)$. Let $\left\{u_{(A)}\right\}=\left\{T, N, B_{1}, B_{2}\right\}$ and $\left\{\bar{u}_{(A)}\right\}=\left\{\bar{T}, \bar{N}, \bar{B}_{1}, \bar{B}_{2}\right\}$, where $A=1,2,3,4$ be the Serret-Frenet tetrads at $s_{0} \in I$ of $r(\alpha(s))$ and $\bar{\alpha}(s)$, respectively. Now, the two Serret-Frenet tetrads of $r(\alpha(s))$ and $\bar{\alpha}(s)$ satisfy the equations

$$
\left[\begin{array}{c}
T^{\prime} \\
N^{\prime} \\
B_{1}^{\prime} \\
B_{2}^{\prime}
\end{array}\right]=\left[\begin{array}{cccc}
0 & k & 0 & 0 \\
k & 0 & \tau & 0 \\
0 & -\tau & 0 & \sigma \\
0 & 0 & -\sigma & 0
\end{array}\right]\left[\begin{array}{l}
T \\
N \\
B_{1} \\
B_{2}
\end{array}\right]
$$


and

$$
\left[\begin{array}{c}
\bar{T}^{\prime} \\
\bar{N}^{\prime} \\
\bar{B}_{1}^{\prime} \\
\bar{B}_{2}^{\prime}
\end{array}\right]=\left[\begin{array}{cccc}
0 & k & 0 & 0 \\
k & 0 & \tau & 0 \\
0 & -\tau & 0 & \sigma \\
0 & 0 & -\sigma & 0
\end{array}\right]\left[\begin{array}{l}
\bar{T} \\
\bar{N} \\
\bar{B}_{1} \\
\bar{B}_{2}
\end{array}\right]
$$

This can be written in a more compact form as

$$
\begin{gathered}
\frac{\mathrm{d} u_{(A)}}{\mathrm{d} s}=\sum_{A}^{\cdots B} a_{A B} u_{(B)} \\
\frac{\mathrm{d} \bar{u}_{(A)}}{\mathrm{d} s}=\sum_{A}^{\cdots B} \bar{a}_{A B} \bar{u}_{(B)}
\end{gathered}
$$

where $B=1,2,3,4$, with $\sum_{A}^{\ldots B} a_{A B}$ and $\sum_{A}^{\ldots B} \bar{a}_{A B}$ denoting the elements of the Serret-Frenet matrix. Clearly, the two tetrads $\left\{u_{(A)}(s)\right\},\left\{\bar{u}_{(A)}(s)\right\}$ are related by an equation of the type

$$
\bar{u}_{(A)}(s)=\wedge_{A}^{\ldots B} b_{A B}(s) u_{(B)}(s)
$$

with the elements of the matrix $\wedge(s)$ satisfying the condition

$$
\wedge_{A}^{\cdots B} b_{A B}\left(s_{0}\right)=\delta_{B}^{A}
$$

Since we are assuming that $u_{(A)}^{\alpha}\left(s_{0}\right)=\bar{u}_{(A)}^{\alpha}\left(s_{0}\right)$.

From (12) and (13) we obtain a system of first-order differential equations for the elements of $\wedge(s)$ given by

$$
\begin{aligned}
& \frac{\mathrm{d} \wedge_{A}^{\cdots B} b_{A B}}{\mathrm{~d} s} u_{(B)}+\wedge_{A}^{\cdots B} \sum_{B}^{\cdots D} b_{A B} a_{B D} u_{(D)} \\
& -\sum_{A}^{\cdots C} \wedge_{C}^{\cdots M} \bar{a}_{A C} b_{C M} u_{(M)}=0
\end{aligned}
$$

By assumption, $\sum_{A}^{\ldots, C, D, G}$ are differentiable functions of the proper parameter $s$. From the theory of ordinary differential equations, we know that if we are given a set of initial conditions $\wedge_{A}^{\ldots B} b_{A B}\left(s_{0}\right)$ then the above system admits a unique solution

$$
\left\{\wedge_{A}^{\cdots B} b_{A B}(s)=\wedge_{A}^{\cdots B} b_{A B}(s)\right\}
$$

defined in an open interval $J \subset I$ containing $s_{0}$. On the other hand, it is easily seen that $\wedge_{A}^{\cdots B} b_{A B}(s)=\delta_{B}^{A}$, is a solution of (14). Therefore, we conclude that $\bar{u}_{(A)}(s)=u_{(B)}(s)$.

\section{REFERENCES}

[1] A. E. El-Ahmady and E. Al-Hesiny, "Conditional Retraction of Some Curves in Minkowski 3-Space," International Journal of Applied Mathematics and Statistics, Vol. 32, No. 2, 2012, pp. 39-47.

[2] A. E. El-Ahmady, "The Variation of the Density on Chaotic Spheres in Chaotic Space-Like Minkowski Space
Time," Chaos, Solitons and Fractals, Vol. 31, No. 5, 2007, pp. 1272-1278. doi:10.1016/j.chaos.2005.10.112

[3] A. E. El-Ahmady and E. Al-Hesiny, "The Topological Folding of the Hyperbola in Minkowski 3-Space," The International Journal of Nonlinear Science, Vol. 11, No. 4, 2011, pp. 451-458.

[4] A. E. El-Ahmady and E. Al-Hesiny, "Folding and Differential Equations of Some Curves in Minkowski Space," Life Science Journal, Vol. 9, No. 2, 2012, pp. 579-584.

[5] A. E. El-Ahmady and E. Al-Hesiny, "Folding and Deformation Retract of Hyperhelix," Journal of Mathematics and Statistics, Vol. 8, No. 2, 2012, pp. 241-247. doi:10.3844/jmssp.2012.241.247

[6] A. E. El-Ahmady, "Retraction of Chaotic Black Hole," The Journal of Fuzzy Mathematics, Vol. 19, No. 4, 2011 , pp. 833-838.

[7] A. E. El-Ahmady, "Limits of Fuzzy Retractions of Fuzzy Hyperspheres and Their Foldings," Tamkang Journal of Mathematics, Vol. 37, No. 1, 2006, pp. 47-55.

[8] A. E. El-Ahmady, "The Deformation Retract and Topo Logical Folding of Buchdahi Space," Periodica Mathematica Hungarica, Vol. 28, No. 1, 1994, pp. 19-30. doi:10.1007/BF01876366

[9] A. E. El-Ahmady, "Folding and Fundamential Group of Buchdahi Space," Indian Journal of Science and Technology, Vol. 6, No. 1, 2013, pp. 3940-3945.

[10] A. E. El-Ahmady, "On the Fundamental Group and Folding of Klein Bottle," International Journal of Applied Mathematics and Statistics, Vol. 37, No. 6, 2013, pp. 56-64.

[11] A. E. El-Ahmady, "The Geodesic Deformation Retract of Klein Bottle and Its Folding," The International Journal of Nonlinear Science, Vol. 12, No. 3, 2011, pp. 323-330.

[12] A. E. El-Ahmady and E. Al-Hesiny, "On Some Curves in Minkowski 3-Space and Its Deformation Retract," International Journal of Applied Mathematics and Statistics, Vol. 36, No. 6, 2013, pp. 42-53.

[13] G. L. Naber, "Topology, Geometry and Gauge Fields, Foundations," Springer-Verlage, New York, Berlin, 2011.

[14] M. Reid and B. Szendroi, "Geometry and Topology," Cambridge University Press, Cambridge, New York, 2005. doi:10.1017/CBO9780511807510

[15] M. Reid and B. Szendroi, "Topology and Geometry," Cambridge University Press, New York, 2005. doi:10.1017/CBO9780511807510

[16] M. Arkowitz, "Introduction to Homotopy Theory," Springer-Village, New York, 2011.

[17] J. Strom, "Modern Classical Homotopy Theory," American Mathematical Society, 2011.

[18] J. M. Lee, "Introduction to Topological Manifolds," Springer-Verlage, New York, 2011. doi:10.1007/978-1-4419-7940-7

[19] R. Lopez, "Differential Geometry of Curves and Surfaces in Lorentz-Minkowski Space," Instituto de Matematica e Estatistica, University of Sao Paulo, Sao Paulo, 2008. 